\title{
Reciprocal Polynomials Having Small Measure
}

\begin{abstract}
By David W. Boyd*
Abstract. The measure of a monic polynomial is the product of the absolute value of the roots which lie outside and on the unit circle. We describe an algorithm, based on the root-squaring method of Graeffe, for finding all polynomials with integer coefficients whose measures and degrees are smaller than some previously given bounds. Using the algorithm, we find all such polynomials of degree at most 16 whose measures are at most 1.3. We also find all polynomials of height 1 and degree at most 26 whose measures satisfy this bound. Our results lend some support to Lehmer's conjecture. In particular, we find no noncyclotomic polynomial whose measure is less than the degree 10 example given by Lehmer in 1933.
\end{abstract}

1. Introduction. The measure $M(P)$ of a polynomial $P(x)=a_{0} x^{n}+\cdots+a_{n}$ with $a_{0} \neq 0$ with zeros $\alpha_{1}, \ldots, \alpha_{n}$ is defined by [8, p. 5]

$$
M(P)=\left|a_{0}\right| \prod_{i=1}^{n} \max \left(\left|\alpha_{i}\right|, 1\right)=\exp \left\{\int_{0}^{1} \log \left|P\left(e^{2 \pi i t}\right)\right| d t\right\} .
$$

If $P$ has integer coefficients, as we assume unless otherwise stated, Kronecker's theorem tells us that if $M(P)=1$ and $a_{n} \neq 0$, then $P$ is cyclotomic. Lehmer [7] raised the question of whether there might be a constant $\epsilon_{0}>0$ independent of $n$ so that, if $P$ is not cyclotomic, then $M(P) \geqslant 1+\epsilon_{0}$.

Smyth [12] has proved that if $P$ is nonreciprocal, then $M(P) \geqslant \theta_{0}$, where $\theta_{0}=$ $1.3247 \ldots$ is the smallest Pisot-Vijayaraghavan number. For reciprocal $P$, the question is still open, although Dobrowolski [5] has recently proved that

$$
M(P) \geqslant 1+\frac{1}{1200}\left(\frac{\log \log n}{\log n}\right)^{3},
$$

unless $P$ is cyclotomic. Surveys of related results have been given by Stewart [13] and the author [3]. In [3], we used Lehmer's notation $\Omega(P)$ for $M(P)$ and (unfortunately) $M(P)$ for $\max \left|\alpha_{i}\right|$. The notation adopted here is due to Mahler [8] .

In [7, p. 477], Lehmer states that, "we have not made an examination of all 10th degree symmetric polynomials, but a rather intensive search has failed to reveal a better polynomial than

$$
x^{10}+x^{9}-x^{7}-x^{6}-x^{5}-x^{4}-x^{3}+x+1, \Omega=1.176280821 .
$$

All efforts to find a better equation of degree 12 and 14 have been unsuccessful".

Received May 21, 1979; revised March 24, 1980.

1980 Mathematics Subject Classification. Primary 12A20; Secondary 12-04, 26C05, 12 A 15. of Canada.

* Supported in part by a grant from the Natural Sciences and Engineering Research Council 
In view of the current interest in Lehmer's conjecture and urged on by $\mathrm{C}$. $\mathrm{L}$. Stewart, we recently carried out an exhaustive search of polynomials of small degree $(n \leqslant 16)$ for those of small measure (generally $M \leqslant 1.3$ ), and in addition a search of all polynomials of height 1 up to $n=26$. (In this paper, the height $H(P)$ is defined by $\left.H(P)=\max \left|a_{i}\right|.\right)$ We also investigated a number of families of polynomials as described in Section 5. In spite of this extensive investigation, we found no better polynomial than Lehmer's 1933 example.

Because of Smyth's result, we were able to restrict consideration to reciprocal polynomials, although it should be pointed out that the structure of the set $\{M(P)$ : $P$ nonreciprocal $\}$ is far from being well understood. With $M(P)<2$, we have $\left|a_{0}\right|=1$ and hence we may assume that $a_{0}=a_{n}=1$. Using the symmetry $x \rightarrow-x$, we may also assume that $a_{1} \geqslant 0$. If $P(x)=Q\left(x^{s}\right)$, then $M(P)=M(Q)$, so we can omit $P$ of this form with $s>1$.

For any fixed $M$ and $n$, the set of $P$ with degree $n$ and $M(P) \leqslant M$ is clearly finite. For example [8, p. 7],

$$
\left|a_{k}\right| \leqslant\left(\begin{array}{l}
n \\
k
\end{array}\right) M .
$$

However, the number of reciprocal $P$ satisfying (2) with, say, $n=10, M=1.3, a_{0}=1$, and $a_{1} \geqslant 0$ is about $1.5 \times 10^{11}$ so a direct calculation of $M(P)$ for all such $P$ is out of the question. Using Newton's identities, a smaller set results, as described in Section 3 (c), but even for relatively small $n$ a more elaborate algorithm is needed.

Our approach is based on the root-squaring method of Graeffe, described in most numerical analysis texts and in complete detail by Bareiss [1]. The efficiency of our algorithm depends to a considerable extent on certain improvements of (2) given in Section 2. However, the basic idea can be illustrated using (2), and the following consequence of (1) and (2), [8, pp. 7-8]: Let $L(P)=\sum_{k=0}^{n}\left|a_{k}\right|$ be the length of $P$, then

$$
M(P) \leqslant L(P) \leqslant 2^{n} M(P) .
$$

Let $P_{m}(x)=a(0, m) x^{n}+\cdots+a(n, m)$ be the monic polynomial whose roots are the $2^{m}$ th powers of the roots of $P$. As is well known,

$$
a(k, m)=(-1)^{k} a(k, m-1)^{2}
$$

$$
+2 \sum_{j=1}^{\min (k, n-k)}(-1)^{j+k} a(k-j, m-1) a(k+j, m-1),
$$

so $P_{1}, P_{2}, \ldots$ are easily generated recursively. Since evidently $M\left(P_{m}\right)=M(P)^{2^{m}}$, the requirement $M(P) \leqslant M$ and (2) imply that

$$
|a(k, m)| \leqslant\left(\begin{array}{l}
n \\
k
\end{array}\right) M^{2^{m}} .
$$

Thus, if one begins with an initial set $R_{0}$ of polynomials (all those satisfying (2), say), one obtains in succession sets $R_{0} \supset R_{1} \supset R_{2} \supset \cdots$, where $R_{m}$ consists of those polynomials in $R_{m-1}$ satisfying (5). Now (3) implies that

$$
M(P)=M\left(P_{m}\right)^{2-m} \leqslant L\left(P_{m}\right)^{2^{-m}} \leqslant 2^{n / 2^{m}} M(P),
$$


so that $R_{m}$ contains only polynomials with measures $\leqslant 2^{n / 2^{m}} M \quad$ Thus $R_{\infty}=\bigcap R_{m}$ is exactly the set $R_{0} \cap\{M(P) \leqslant M\}$.

In fact one stops the process for a moderately small value of $m=m^{*}$ and simply computes $M(P)$ for all $P$ in $R_{m}$ * from (1). Although (6) shows that $\lim L\left(P_{m}\right)^{2^{-m}}$ $=M(P)$, we have found that the direct computation of $M(P)$ by finding the zeros of $P$ and using (1) is a more effective way of calculating an accurate value of $M(P)$. The advantage of (6) is that it gives reasonably good bounds on $M(P)$ by a small amount of computation.

An additional feature of this approach is that if $P$ is cyclotomic, then $P_{m}=$ $P_{m+1}$ as soon as $2^{m-1}>n$ (see Section 3(b)). Thus the cyclotomic polynomials may be detected in a small number of steps.

Timings are given in Section 4. Initially we hoped to extend our exhaustive search to $n=20$ but eventually settled for $n \leqslant 16$. The case $n=18$ is feasible, but potentially expensive, so this has been deferred in the hope of future theoretical progress or else an improved algorithm.

The search among polynomials with $H(P)=\max \left|a_{i}\right|=1$ is considerably less expensive. It is known that, for any $P$ with $M(P)<2$, there is a $Q$ with integer coefficients with $H(P Q)=1$. It has been our experience that $Q$ can be taken to be cyclotomic and of fairly small degree relative to $n$. The proof of a quantitative result of this nature would greatly enhance the value of the lists in Section 6 for $18 \leqslant n \leqslant 26$.

2. The Basic Inequalities. In this section, $P$ is any polynomial with complex coefficients. We shall derive a number of inequalities for $\left|a_{k}\right|$ which depend on $M(P)$.

We begin by recalling the notion of Schur convexity [10, pp. 167-168]. Suppose that $x=\left(x_{1}, \ldots, x_{n}\right)$ is a vector with real components and that $x_{1}^{*}, \ldots, x_{n}^{*}$ denote $x_{1}, \ldots, x_{n}$ arranged in decreasing order. If $y=\left(y_{1}, \ldots, y_{n}\right)$, we write $x \prec y$ provided that

$$
\sum_{i=1}^{k} x_{i}^{*} \leqslant \sum_{i=1}^{k} y_{i}^{*}, \quad k=1, \ldots, n,
$$

with equality holding if $k=n$. A function $F(x)$ is said to be Schur convex if $x \prec y$ implies $F(x) \leqslant F(y)$.

LEMma 1 (SCHUR, OSTROWSKI [10, p. 168]). If $F$ is differentiable, then it is Schur convex if and only if $\left(x_{i}-x_{j}\right)\left(F_{i}-F_{j}\right) \geqslant 0$, for all $i$ and $j$, where $F_{i}$ denotes the partial derivative with respect to $x_{i}$.

LEMmA 2. Let $\sigma_{k}\left(\alpha_{1}, \ldots, \alpha_{n}\right)$ be the $k$ th elementary symmetric function of $\alpha_{1}, \ldots, \alpha_{n}$. Then $F(x)=\sigma_{k}\left(e^{x_{1}}, \ldots, e^{x_{n}}\right)$ is a Schur convex function of $x$.

Proof. $F_{i}-F_{j}=\left(e^{x_{i}}-e^{x_{j}}\right) A$, where $A$ is a nonnegative function of the remaining variables.

Lemma 3. Let $P(x)=a_{0} x^{n}+\cdots+a_{n}$ satisfy $M(P) \leqslant M$. Then

$$
\left|a_{k}\right| \leqslant\left(\begin{array}{l}
n-2 \\
k-1
\end{array}\right) M+\left(\begin{array}{l}
n-2 \\
k-2
\end{array}\right)\left|a_{n}\right|+\left(\begin{array}{c}
n-2 \\
k
\end{array}\right)\left|a_{0}\right|+\left(\begin{array}{l}
n-2 \\
k-1
\end{array}\right)\left|a_{0} a_{n}\right| M^{-1}
$$


Proof. Assume for the moment that $a_{n} \neq 0$. Number the roots of $P$ so that $\left|\alpha_{1}\right| \geqslant \cdots \geqslant\left|\alpha_{m}\right| \geqslant 1>\left|\alpha_{m+1}\right| \geqslant \cdots \geqslant\left|\alpha_{n}\right|>0$. Define $x_{i}=\log \left|\alpha_{i}\right|$. Then

$$
\begin{aligned}
x_{1}+\cdots+x_{m} & =\log \left(M(P) /\left|a_{0}\right|\right) \leqslant \log \left(M /\left|a_{0}\right|\right), \\
x_{m+1}+\cdots+x_{n} & =\log \left(\left|a_{n}\right| / M(P)\right) \geqslant \log \left(\left|a_{n}\right| / M\right) .
\end{aligned}
$$

If we define $y_{1}=\log \left(M /\left|a_{0}\right|\right), y_{n}=\log \left(\left|a_{n}\right| / M\right)$, and $y_{i}=0$ otherwise, then it is easy to check that $x \prec y$. Hence, by Lemma 2,

$$
\sigma_{k}\left(\left|\alpha_{1}\right|, \ldots,\left|\alpha_{n}\right|\right) \leqslant \sigma_{k}\left(M /\left|a_{0}\right|, 1, \ldots, 1,\left|a_{n}\right| / M\right),
$$

from which (7) is immediate.

Using continuity, the assumption $a_{n} \neq 0$ can now be dropped.

Remarks. 1. The inequality (7) is uninformative if $k=0$ or $n$, but in these cases $\left|a_{0}\right| \leqslant M$ and $\left|a_{n}\right| \leqslant M$. For our purposes $a_{0}=a_{n}=1$, so (7) improves (2) considerably, especially for large $M$. Note that (2) is sharp only if $M=1$, while (7) is sharp for all $M$, with equality for $P(x)=(x+M)(x+1)^{n-2}\left(x+M^{-1}\right)$.

2. It is often possible to obtain information concerning $\max \left|\alpha_{i}\right|$ and $\min \left|\alpha_{i}\right|$, (see e.g. Lemma 6 or [9, Chapter 7]). Lemma 2 allows us to use this information very easily, as the next result illustrates:

Lemma 4. Let $P$ satisfy $M(P) \leqslant M$. Suppose in addition that $\left|\alpha_{1}\right|=\max \left|a_{i}\right| \leqslant$ $a<M /\left|a_{0}\right|$, and that $\left|\alpha_{n}\right|=\min \left|\alpha_{i}\right| \geqslant c>a_{n} / M$. Define integers $s, t$ and real numbers $b, d$ with $1 \leqslant b<a, 1 \leqslant d<c$ by

$$
a^{s} b=M /\left|a_{0}\right| \text { and } \quad c^{t} d=\left|a_{n}\right| / M
$$

Then

$$
\left|a_{k}\right| \leqslant\left|a_{0}\right| \sigma_{k}(a, \ldots, a, b, 1, \ldots, 1, d, c, \ldots, c),
$$

where $a, c$, and 1 are repeated respectively $s, t$, and $n-s-t-2$ times.

Proof. Analogous to Lemma 3 except that $y$ is defined by $y_{1}=\cdots=y_{s}=$ $\log a, y_{s+1}=\log b, y_{n-t}=\log d, y_{n-t+1}=\cdots=y_{n}=\log c$, and $y_{i}=0$ otherwise. Obvious modifications are of course made if $n-s-t-2<0$.

Lemma 5. If $P$ is a reciprocal polynomial with $a_{0}=a_{n}=1$, for which $M(P) \leqslant$ $M$ and $\max \left|\alpha_{i}\right| \leqslant M^{1 / 2}$, then

$$
\begin{aligned}
\left|a_{k}\right| \leqslant & \left(M+4+M^{-1}\right)\left(\begin{array}{l}
n-4 \\
k-2
\end{array}\right)+2\left(M^{1 / 2}+M^{-1 / 2}\right)\left(\begin{array}{l}
n-4 \\
k-3
\end{array}\right)+\left(\begin{array}{l}
n-4 \\
k-1
\end{array}\right) \\
& +\left(\begin{array}{l}
n-4 \\
k-4
\end{array}\right)+\left(\begin{array}{c}
n-4 \\
k
\end{array}\right) .
\end{aligned}
$$

Proof. A special case of Lemma 4.

LEMMA 6. Let $P$ be a reciprocal polynomial with real coefficients and $a_{0}=$ $a_{n}=1$. Suppose in addition that all real roots of $P$ are positive. If $a_{1} \geqslant n-4$, then $\left|\alpha_{1}\right|=\max \left|\alpha_{i}\right| \leqslant M(P)^{1 / 2}$. 
Proof. If $\alpha_{1}$ is not real, then $\bar{\alpha}_{1}$ is also a root of $P$ so $\left|\alpha_{1}\right|^{2} \leqslant M$. Thus we may assume $\alpha_{1}$ is real, hence positive, and that $\alpha_{1}>M^{1 / 2}$. Let $\beta_{1}, \ldots, \beta_{2 s}$ be the complex roots of $P$. Then

$$
\prod_{i=1}^{2 s} \max \left(\left|\beta_{i}\right|, 1\right) \leqslant M / \alpha_{1}<M^{1 / 2}
$$

so that $\left|\beta_{i}\right|<M^{1 / 4}$ for all $i$. Hence, by Lemma 4,

$$
\sum \beta_{i} \leqslant \sum\left|\beta_{i}\right|<2\left(M^{1 / 4}+M^{-1 / 4}\right)+n-6 .
$$

Since $P$ is reciprocal, $\alpha_{1}^{-1}$ is also a root of $P$, so

$$
\sum_{\alpha_{i} \text { real }} \alpha_{i} \geqslant \alpha_{1}+\alpha_{1}^{-1}>M^{1 / 2}+M^{-1 / 2}
$$

Combining these inequalities,

$$
-a_{1}=\sum_{i=1}^{n} \alpha_{i}>M^{1 / 2}+M^{-1 / 2}-2 M^{1 / 4}-2 M^{-1 / 4}-(n-6) .
$$

The right member of (13) is an increasing function of $M$ for $M \geqslant 1$, hence (13) implies $a_{1}<n-4$. Thus $a_{1} \geqslant n-4$ implies $\left|\alpha_{1}\right| \leqslant M^{1 / 2}$.

Remarks. 3. The assumption that the real roots are positive is valid for $P_{m}$ if $m \geqslant 1$.

4. A corresponding result is true for nonreciprocal $P$, with $n-4$ replaced by $n-2$.

Lemma 7. Let $\alpha_{1}, \ldots, \alpha_{n}$ be a set of complex numbers closed under complex conjugation. Then

$$
\frac{2}{n}\left(\sum_{k=1}^{n} \alpha_{k}\right)^{2} \leqslant \sum_{k=1}^{n} \alpha_{k}^{2}+\sum_{k=1}^{n}\left|\alpha_{k}\right|^{2}
$$

Proof. Let $\alpha_{k}=\beta_{k} e^{i \theta} k, \beta_{k} \geqslant 0$. Then

$$
\sum \alpha_{k}^{2}+\sum\left|\alpha_{k}\right|^{2}=\sum \beta_{k}^{2}\left(\cos \left(2 \theta_{k}\right)+1\right)=2 \sum \beta_{k}^{2} \cos ^{2} \theta_{k} .
$$

Thus (14) follows from the Cauchy-Schwarz inequality.

Lemma 8. Let $L_{2}^{2}(P)=\left|a_{0}\right|^{2}+\cdots+\left|a_{n}\right|^{2}$. Then

$$
\begin{aligned}
& M(P) \leqslant L_{2}(P) \leqslant\left(\begin{array}{c}
2 n \\
n
\end{array}\right)^{1 / 2} M(P), \\
& M(P)^{2}+\left|a_{0} a_{n}\right|^{2} M(P)^{-2} \leqslant L_{2}^{2}(P) .
\end{aligned}
$$

Proof. The left inequality of (15) is due to Specht. Using the second definition in (1), it is easily seen to be just the arithmetic-geometric inequality for integrals, together with Parseval's relation [9, p. 129]. The right-hand inequality of (15) follows from (2), as observed by Duncan [6]. The inequality (16) is due to Gonçalves, a more direct proof being given by Ostrowski [11] 
3. The Algorithm. We have outlined our basic approach in Section 1. However, there are a number of details to be considered before a workable algorithm is obtained. We shall deal in turn with (a) the root-squaring process, (b) cyclotomic factors, and (c) selection of the initial set $R_{0}$ for exhaustive search.

For the remainder of the paper, $P$ will denote a monic reciprocal polynomial of even degree $n=2 h$ with integer coefficients. Given a set of such polynomials $R_{0}$, we are seeking those which have $M(P) \leqslant M$, where $M$ is a parameter to be specified.

According to Lemma 6 , if $a(1, m) \geqslant n-4$ for any $m \geqslant 1$, then $\left|\alpha_{1}\right| \leqslant$ $M(P)^{1 / 2}$, in which case (12) provides improved estimates over (7). We thus find it useful to associate with $P$ a parameter $s=s(P)$ taking on one of the values 1 or 2 to indicate that $\left|\alpha_{1}\right| \leqslant M(P)^{1 / s}$. Initially $s=1$, but if at some point in the processing of $P$ we determine that $\left|\alpha_{1}\right| \leqslant M(P)^{1 / 2}$, then we set $s=2$.

3(a) Root-Squaring. We regard $n$ and $M$ as fixed and $R_{0}$ as given. Given $P$ in $R_{0}$, we wish to reject $P$ from consideration if $M(P)>M$.

If $M(P) \leqslant M$ and $\left|\alpha_{1}\right| \leqslant M(P)^{1 / s}$, the coefficients $a(k, m)$ of $P_{m}$ satisfy

$$
|a(k, m)| \leqslant b(k, m, s),
$$

where the quantities $b(k, m, s)$ are given by (7) and (12) as

$$
\begin{aligned}
b(k, m, 1)= & \left(\begin{array}{l}
n-2 \\
k-1
\end{array}\right)\left(M^{2^{m}}+M^{-2^{m}}\right)+\left(\begin{array}{l}
n-2 \\
k-2
\end{array}\right)+\left(\begin{array}{c}
n-2 \\
k
\end{array}\right), \\
b(k, m, 2)= & \left(\begin{array}{l}
n-4 \\
k-2
\end{array}\right)\left(M^{2^{m}}+4+M^{-2^{m}}\right)+2\left(\left(\begin{array}{l}
n-4 \\
k-3
\end{array}\right)+\left(\begin{array}{l}
n-2 \\
k-1
\end{array}\right)\right) \\
& \cdot\left(M^{2^{m-1}}+M^{2^{m-1}}\right)+\left(\begin{array}{c}
n-4 \\
k-4
\end{array}\right)+\left(\begin{array}{c}
n-4 \\
k
\end{array}\right) .
\end{aligned}
$$

These can be computed and tabulated once $n$ and $M$ have been specified.

We can then successively compute $a(1, m), \ldots, a(k, m)$, for $m=1,2, \ldots, m^{*}$, using (4). If (17) is violated for any $(k, m)$, we reject $P$. If $a(1, m) \geqslant n-4$ for any $m \geqslant 1$, we set $s=2$. The advantage of this is fairly evident when one considers the behavior of $b(k, m, 2)$ versus $b(k, m, 1)$ as $m \rightarrow \infty$.

In addition to this "rejection criterion", we include, for $m \geqslant m_{1}$, an "acceptance criterion" based on (15). Thus, if $L_{2}\left(P_{m}\right) \leqslant M^{2^{m}}$, then $M(P) \leqslant M$, so we need no further root-squarings. The cost of calculating $L_{2}^{2}\left(P_{m}\right)$ is the same as that of computing $a(m+1, h)$. The choice of $m_{1}$ is discussed in 3(b).

We also accept $P$ if it survives (17) for all $m \leqslant m^{*}$, even though there is the possibility that $M(P)>M$. The best choice of $m^{*}$ depends on the relative sizes of the sets $R_{m}$, which are initially unknown, and on the time needed to compute $P_{m}$ from $P_{m-1}$ relative to the time needed to calculate $M(P)$. To see this, consider the relative advantages of computing $M(P)$ for all $P$ in $R_{m-1}$ versus computing $P_{m}$ for all $P$ in $R_{m-1}$ and then computing $M(P)$ for all $P$ in $R_{m}$.

The choice of $m^{*}$ affects the size of the $a(k, m)$, so we settled on a choice which would allow the $a(k, m)$ to be represented exactly as double-precision reals. This restricted $m^{*} \leqslant 7$ in most cases, but fortunately this proved to be close to the optimal choice for $n \leqslant 16$. 
This is an appropriate point to discuss whether it is really necessary to compute $a(k, m)$ exactly, or whether one could not simply increase the $b(k, m, s)$ by a certain amount to compensate for errors in the $a(k, m)$. The answer hinges on how sensitive $M(P)$ is to small perturbations in the coefficients of $P$, so the following example is perhaps instructive:

Example. Let $P(x)=(x+1)^{2 h}$, and $Q(x)=(x+1)^{2 h}-x^{h}$. Then $M(P)=1$ and $P$ has large coefficients, indeed as large as possible according to (2). $Q$ thus represents a small perturbation of $P$. If $h=30$, for example, the middle coefficient of $P$ is $\left(\begin{array}{l}60 \\ 30\end{array}\right)=$ $1.18 \times 10^{17}>2^{56}$, so $P$ and $Q$ would be identically represented in double precision on the machine used.

On the other hand, it is easy to see that

$$
\begin{aligned}
\log M(Q) & =2 \int_{0}^{1 / 2} \log \left|(z+1)^{2 h}-z^{h}\right| d t \quad(z=\exp (2 \pi i t)) \\
& =2 \int_{0}^{1 / 2} \log \left|\left(z^{1 / 2}+z^{-1 / 2}\right)^{n}-1\right| d t, \quad \text { writing } n=2 h
\end{aligned}
$$

so

$$
\log M(Q) \sim 2 \int_{E} \log \left|(2 \cos \pi t)^{n}\right| d t=n \log \beta,
$$

where $E=\{0 \leqslant t \leqslant 1 / 2: 2 \cos \pi t>1\}=[0,1 / 3)$, and

$$
\beta=\exp \left\{2 \int_{0}^{1 / 3} \log (2 \cos \pi t) d t\right\}=1.381356444 \text {. }
$$

Thus $M(Q) \sim \beta^{n}$. Coincidentally $\beta=\lim _{n \rightarrow \infty} M\left(z^{n}+z+1\right)$ [3] , and so $\beta$ was calculated in [4]. Using estimates given in [4], it is easy to obtain the more precise result that $M(Q)=\beta^{n} e^{-\epsilon} n$, where $0<\epsilon_{n}<4 / n$.

For example, if $n=64$, then $M(Q) \sim 9.54 \times 10^{8}$, contrasting markedly with $M(P)=1$. Of course $P$ is very "ill-conditioned" because of the multiple root at -1 . However, observe that if $P_{0}=x^{64}+1$, which is a reasonably well-conditioned polynomial, then $P_{6}=(x+1)^{64}$. Even if we were in a situation where $Q=Q_{6}$ for some initial $Q_{0}$, we would still have $M\left(Q_{0}\right) \sim \beta=1.38 \ldots$ which is still not very satisfactory.

3(b) Cyclotomic Factors. It is obvious that many of the polynomials $P$ of degree $n$ with $M(P) \leqslant M$ will be reducible since if $S, T$ have $M(S) \leqslant M, M(T)=1$, and $\operatorname{deg} S$ $+\operatorname{deg} T=n$, then $P=S T$ has $M(P) \leqslant M$. Assuming that we have already computed $M(S)$, it would be inefficient to again compute $M(P)$. Similar remarks apply if $P$ is cyclotomic.

The method of recognizing those $P$ with cyclotomic factors depends to a certain extent on $R_{0}$. Let us begin by observing the effect of root-squaring on cyclotomic polynomials. Suppose that $Q=F_{2{ }^{k}}$ is the irreducible cyclotomic polynomial whose roots are the primitive $2^{k} q$ th roots of unity, with $q$ odd, so $\operatorname{deg} Q=2^{k-1} \phi(q)$, if $k \geqslant 1$, and $=\phi(q)$, if $k=0$. Clearly $Q_{m}=F_{r}^{s}$, where $r=2^{k-m} q$ and $s=2^{m}$ if $m \leqslant k-1$, while $r=q$ and $s=2^{k-1}$ if $m \geqslant k$. Hence, after $m \geqslant k$ root-squarings, a cyclotomic factor $F_{{ }^{k}}$ of $P$ stabilizes as a factor $F_{q}$ of $P_{m}$ with multiplicity $2^{k-1}$. Since $\operatorname{deg} F_{2 k_{q}} \leqslant \operatorname{deg} P=n$, we have $2^{k-1} \leqslant n$, so after at most $m_{1}=\left[\log _{2} n\right]+1$ 
root-squarings, all cyclotomic factors are of the form $F_{q}$ with $q$ odd.

As a consequence, if $P$ is cyclotomic, then $P_{m}=P_{m+1}$ for some $m \leqslant m_{1}$, and hence $P$ is detectable by at most $m_{1}+1$ root-squarings.

If $R_{0}$ is chosen for exhaustive search, then we can afford to ignore any reducible polynomials in $R_{0}$, since we naturally intend to search in order of increasing $n$. Cyclotomic factors can be detected simply by testing $P_{m_{1}}$ for factors $F_{q}$ with $q$ odd. Since cyclotomic $P$ are detected by the method of the previous paragraph, we can assume that $P$ is not cyclotomic and hence that $\operatorname{deg} F_{q} \leqslant n-n_{0}$, where $n_{0}$ is the smallest degree for which there is a polynomial $Q$ having $M(Q) \leqslant M$. For example, if $M=$ 1.3 , then $n_{0}=8$, and this fact is known before we attempt $n=10$.

In our implementation, we decided to check only for the factors $F_{1}$ and $F_{p}$ where $p$ is prime, since in this case there is a very easy criterion for $F_{p}$ to divide $P$. We begin by computing $P_{m_{1}}(1)=t$. If $t=0$, then $F_{2 k}$ divides $P$ for some $k$, so $P$ is discarded. Since $F_{p}(1)=p$, a necessary condition for $F_{2 k_{p}}$ to divide $P$ is that $p \mid t$. If this holds, then we test whether $F_{p}$ divides $P_{m_{1}}$ using the following:

Lemma 9. Let $P=a_{0} x^{n}+\cdots+a_{n}$ and let $p$ be prime. Then $F_{p}$ divides $P$ if and only if $c_{0}=c_{1}=\cdots=c_{p-1}$, where

$$
c_{i}=\sum_{k \equiv i(\bmod p)} a_{k}
$$

Proof. $F_{p}$ divides $P$ if and only if $x^{p}-1$ divides $(x-1) P$.

Since $a_{k}=a_{n-k}$, it follows that $c_{i}=c_{n-i}$, so only about half of the $c_{i}$ need be calculated. For example, if $n=16$ and $p=5$, then $c_{0}=c_{16}=c_{1}$ and $c_{2}=c_{4}$, so one need only check that $c_{0}=c_{2}=c_{3}$.

This approach is not appropriate when $R_{0}$ consists of all $P$ of degree $n$ and $H(P)$ $=1$, since it would reject those $P$ with a factor $P_{0}$ having $H\left(P_{0}\right)>1$. Instead, we adopted the following method. Note that if $P$ has exactly $\nu=\nu(P)$ roots outside the unit circle, then $|a(\nu, m)|^{2-m} \rightarrow M(P)$. In fact, $a(\nu, m)$ is the trace of the polynomial of degree $\left(\begin{array}{l}n \\ \nu\end{array}\right)$ whose roots are $\left(\alpha_{i_{1}} \cdots \alpha_{i_{\nu}}\right)^{2 m}$, so

$$
|| a(\nu, m)\left|-M(P)^{2^{m}}\right| \leqslant\left(\left(\begin{array}{l}
n \\
\nu
\end{array}\right)-1\right) M^{2^{m}}\left|\alpha_{\nu}\right|^{-2^{m}}
$$

If $P$ is in $R_{m}$, then $|a(\nu, m)|^{2-m}$ provides an approximation to $M(P)$. Of course we do not usually know $\nu(P)$. Suppose, however, that for some $M_{1}>M$, we maintain a list of all previously discovered $P_{0}$ with $M\left(P_{0}\right) \leqslant M_{1}$ and $\operatorname{deg} P_{0}<n$, arranged in order of $\nu\left(P_{0}\right)$, then $M\left(P_{0}\right)$. Then, for $m=m^{*}$, we need only search the list (by binary search) for a $P_{0}$ with $\nu\left(P_{0}\right)=k$ and $M\left(P_{0}\right) \simeq|a(k, m)|^{2-m}$, for $k=s, \ldots, h$. If a suitable $P_{0}$ is found, with $P_{0}(x)=Q\left(x^{r}\right)$, then we need only test whether $Q\left( \pm x^{r}\right)$ divides $P$.

If this is successful, we avoid solving $P(x)=0$. If $\nu(P)=1$ or $\nu(P)=2$ and the roots outside the unit circle are complex conjugates, then the approximation to $M(P)$ is sufficiently accurate that $P_{0}$ is usually located. In other cases, the search may fail and then $P(x)=0$ is solved needlessly, but on balance this test has proved to be worthwhile. 
3(c) Selection of $R_{0}$ for Exhaustive Search. Recall Newton's identities:

$$
S_{k}+a_{1} S_{k-1}+\cdots+a_{k-1} S_{1}+k a_{k}=0,
$$

where $S_{k}=\alpha_{1}^{k}+\cdots+\alpha_{n}^{k}$. By Lemmas 3 and 5, we have

$$
S_{k} \leqslant[c(k, s)]
$$

where

$$
\begin{aligned}
& c(k, 1)=M^{k}+n-2+M^{-k}, \\
& c(k, 2)=2 M^{k / 2}+n-4+2 M^{-k / 2} .
\end{aligned}
$$

Thus, if $a_{1}, \ldots, a_{k-1}$ are specified, then (21) shows that $a_{k}$ is restricted to an interval of length $2[c(k, s)] / k$. For example, if $n=10, M=1.3$, we find from (22) and (21) that we need only examine at most 32560 polynomials, a considerable improvement over the number $1.5 \times 10^{11}$ suggested before.

Applying Lemma 6 to the polynomial with roots $\alpha_{1}^{k}, \ldots, \alpha_{n}^{k}$, which is valid if $k$ is even, shows that $-S_{k} \geqslant n-4$ for $k$ even implies $s=2$, a fact which can be used in all further processing of $P$. Lemma 7 provides the additional bounds

$$
-S_{2 k} \leqslant-2 S_{k}^{2} / n+c(2 k, s)
$$

where $[c(2 k, s)]$ is not used since $\Sigma\left|\alpha_{i}\right|^{2 k}$ need not be an integer. This bound is very restrictive if $\left|S_{k}\right|$ is near $n$.

Since $P$ is reciprocal, the conditions (22) for $k>h$ provide extra conditions on $a_{k}, k \leqslant h$. It was found to be advantageous to use one of these explicitly: using $a_{h-1}$ $=a_{h+1}$ and combining (21) for $k=h$ and $k=h+1$, we find

$$
\begin{aligned}
(h+1) a_{1} a_{h}= & -a_{1}\left(a_{1} S_{h-1}+\cdots+a_{h-1} S_{1}\right) \\
& +\left(a_{2} S_{h-1}+\cdots+a_{h-1} S_{2}\right)+S_{h+1},
\end{aligned}
$$

which, if $a_{1}, \ldots, a_{h-1}$ are given, and $a_{1} \neq 0$, confines $a_{h}$ to an interval of length $2[c(h+1, s)] /(h+1) a_{1}$, often determining $a_{h}$ uniquely if $a_{1} \geqslant 2$.

In addition to all the above, the bound $\left|a_{k}\right| \leqslant b(k, 0, s)$ is occasionally a further restriction.

It should be clear how $R_{0}$ is constructed. In principle, one constructs each $P$ in $R_{0}$ individually then processes it as in 3(a) and 3(b). We in fact allowed the processes of 3(c) and 3(a) to interact somewhat in that the $a(k, m)$ were calculated as soon as enough information was available to determine them. For example, if $n=16$, then $a_{1}, \ldots, a_{6}$ already determine $a(k, 1)$ for $k=1,2,3$ and $a(1,2)$ independent of $a_{7}$ and $a_{8}$. Thus it may be possible to reject all $P$ with a certain initial segment of coefficients.

4. Discussion of the Polynomials Found. For even $n \leqslant 16$, all reciprocal polynomials of degree $n$ with measures $\leqslant M$ were found, where $M=2$ for $n=4,6,8$ and $10, M=1.5$ for $n=12$ and 14 , and $M=1.3$ for $n=16$. An idea of the computation 
involved is given by the CPU time in seconds on the AMDAHL $470 \mathrm{~V} / 6-\mathrm{II}$ at the University of British Columbia:

\begin{tabular}{rrrc}
\hline$n$ & $M$ & time & number found \\
\hline 10 & 1.3 & 1.235 & 7 \\
10 & 2.0 & 11.694 & 185 \\
12 & 1.5 & 16.108 & 43 \\
14 & 1.5 & 191.245 & 93 \\
16 & 1.3 & 827.884 & 14
\end{tabular}

For $n=10, \ldots, 26$, all polynomials with $H(P)=1$ and $M(P) \leqslant 1.3$ were found. The times in seconds for $n=16,18,20,22,24$, and 26 are 4.496, 13.799, 42.010, $131.236,396.384$, and 1232.219 respectively.

The tables in Section 6 contain the noncyclotomic factors of a subset of the polynomials just mentioned, normalized so that the first nonzero $a_{k}$ with $k$ odd has $a_{k}>0$. The column "number found" in the above table gives the total number of polynomials normalized in this manner which would appear in a complete list.

Using the fact that $M\left(P_{1} P_{2}\right)=M\left(P_{1}\right) M\left(P_{2}\right)$, and the fact that we know all reciprocal polynomials of degree at most 16 and of sufficiently small measure, it is easy to decide whether any of the polynomials are reducible. From our data, we can rule out possible factorizations of the form $P=P_{1} P_{2}$ with $P_{1}$ and $P_{2}$ being reciprocal and noncyclotomic. Using Smyth's result on nonreciprocal polynomials, we can rule out nonreciprocal factors $P_{0}$ of $P$, provided $M(P)>\theta_{0}^{2}=1.7548 \ldots$, since if a nonreciprocal $P_{0}$ divides $P$, then so does its reciprocal. It turns out that, after cyclotomic factors have been removed, the only reducible polynomials which remain have $\nu(P)=$ $n / 2$ and factor in the form $P_{0} P_{0}^{*}$ where $P_{0}$ is nonreciprocal and $P_{0}^{*}$ is the reciprocal of $P_{0}$ normalized to be monic.

Among the $P$ of small degree there are a number of remarkable occurrences. For example, the following polynomials, all with $\nu(P)=2$, have the same measures:

(i) $M=1.7467934983, \quad P_{1}=122212221, \operatorname{deg} P_{1}=6$, $P_{2}=\begin{array}{llllllllll}1 & 1 & -1 & -1 & 1 & -1 & -1 & 1 & 1, \operatorname{deg} P_{2}=8,\end{array}$

(ii) $M=1.7709445842, P_{1}=\begin{array}{lllllll}1 & 1 & 0 & -2 & 0 & 1 & 1, \operatorname{deg} P_{1}=6 \text {, }\end{array}$

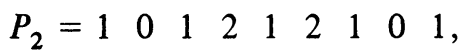
$\begin{array}{lllllllllll}P_{3} & =1 & 2 & 1 & -2 & -3 & -2 & 1 & 2 & 1\end{array}$, $\operatorname{deg} P_{2}=\operatorname{deg} P_{3}=8$,

(iii) $M=1.8789962005, P_{1}=\begin{array}{llllllllllll}1 & 1 & -1 & -3 & 1 & 3 & 1 & -3 & -1 & 1 & 1 & 1\end{array}$

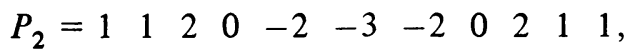

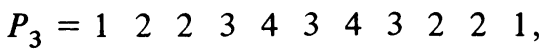


(iv) $M=1.4986652983, \quad P_{1}=\begin{array}{lllllllllllllll}1 & 1 & -1 & -1 & 2 & 0 & -3 & 0 & 2 & -1 & -1 & 1 & 1 \text {, }\end{array}$ $P_{3}=\begin{array}{llllllllllllll}1 & 2 & 2 & 1 & -1 & -3 & -3 & -3 & -1 & 1 & 2 & 2 & 1\end{array}$,

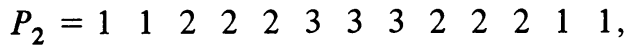

all of degree 12 ,

where we have used a notation whose meaning should be obvious; or see [2].

The triples of the same degree in (iii) and (iv) are most easily explained. If one writes $P_{(3)}$ for the polynomial whose roots are the cubes of the roots of $P$, then it can be verified that, in either (iii) or (iv), each $P_{k}$ has the same $P_{(3)}$ up to the symmetry $x \rightarrow-x$. In each case, if $\omega$ is a primitive cube root of unity, then

$$
P_{1}(\omega x) P_{1}\left(\omega^{2} x\right)=P_{2}(x) P_{3}(-x),
$$

so the surprise is simply that the left member of (27) is reducible over the rationals.

The explanation of (i) and (ii) is somewhat more involved. We first observe that if $\nu(P)=2$, then $M(P)$ is the largest root of the polynomial $Q$ whose roots are $\alpha_{i} \alpha_{j}$, with $i<j$, so $\operatorname{deg} Q=\left(\begin{array}{c}n \\ 2\end{array}\right)$. When $P$ is reciprocal, $h=n / 2$ of the roots of $Q$ are equal to $\alpha_{i} \alpha_{i}^{-1}=1$, hence $M(P)$ is a root of $P^{[2]}(x)=Q(x) /(x-1)^{h}$, the 2 nd compound of $P$, which has degree $n(n-2) / 2$. Note that for $n=6,8$ the degree of $P^{[2]}$ is 12 or 24 , respectively.

A simple calculation then shows that, in example (i),

$$
P_{2}^{[2]}(x)=P_{1}^{[2]}(x) P_{1}(x)^{2}
$$

while, in example (ii),

$$
P_{2}^{[2]}(x)=P_{3}^{[2]}(x)=P_{1}^{[2]}(x) P_{1}(i x) P_{1}(-i x) .
$$

In example (ii), there is additionally

$$
P_{2}(x) P_{2}(-x)=P_{3}(i x) P_{3}(-i x) .
$$

One moral of the above is that $M(P)$ does not determine $P$ up to the obvious transformations $P(x) \rightarrow P\left( \pm x^{s}\right)$. In fact, it is quite possible for a nonreciprocal $P_{1}$ to have the same mesaure as a reciprocal $P_{2}$. For example, if

$$
P_{1}(x)=(x-\alpha)(x-\bar{\alpha})(x-\beta)(x-\bar{\beta})
$$

has $|\alpha|>1>|\beta|=|\alpha|^{-1}$, but is nonreciprocal, so $\beta \neq \alpha^{-1}, \bar{\alpha}^{-1}$, then $M\left(P_{1}\right)=$ $|\alpha|^{2}$ is the unique root outside the unit circle of a reciprocal polynomial $P_{1}^{[2]}$ of

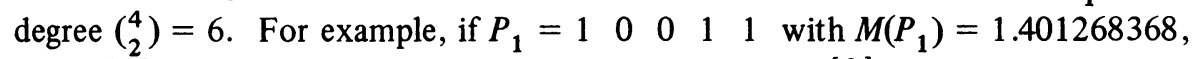
then $P_{1}^{[2]}=\begin{array}{lllllll}1 & 0 & -1 & -1 & -1 & 0 & 1\end{array}$, so that $P_{2}(x)=P_{1}^{[2]}(-x)$ has $M\left(P_{2}\right)=M\left(P_{1}\right)$ as we verify from our list. This explains the following coincidence of measures:

(v) $M=1.9635530390=(1.4012683679)^{2}$, attained for

$$
\begin{aligned}
& P_{3}=12-1-3-121, \quad n=6, \nu\left(P_{3}\right)=1, \text { and } \\
& P_{4}=110131011, \quad n=8, \nu\left(P_{4}\right)=4 .
\end{aligned}
$$

For here we have $P_{3}(x)=P_{2}\left(x^{1 / 2}\right) P_{2}\left(-x^{1 / 2}\right)$, while $P_{4}(x)=P_{1}(x) P_{1}^{*}(x)$. 
5. Nonexhaustive Search. As was mentioned in [3], the set $\{M(P): P$ nonreciprocal \} has at least two limit points smaller than $\theta_{0}=1.3247 \ldots$, namely

$$
\begin{aligned}
\lambda_{1} & =\exp \left\{\int_{0}^{1} \int_{0}^{1} \log \left|\zeta+\zeta z^{-1}+z+1+z^{-1}+\zeta^{-1} z+\zeta^{-1}\right| d s d t\right\} \\
& =1.255425 \ldots \quad\left(\zeta=e^{2 \pi i s}, z=e^{2 \pi i t}\right),
\end{aligned}
$$

and

$$
\lambda_{2}=\exp \left\{\int_{0}^{1} \int_{0}^{1} \log \left|\zeta+z+1+z^{-1}+\zeta^{-1}\right| d s d t\right\}=1.285734 \ldots
$$

These are the unique limit points of the following sets of polynomials:

$$
P_{1}(x)=x^{2 h}+a x^{2 h-m}+b x^{h+m}+c x^{h}+b x^{h-m}+a x^{h}+1,
$$

and

$$
P_{2}(x)=x^{2 h}+a x^{h+m}+b x^{h}+a x^{h-m}+1,
$$

respectively, where $m$ and $h$ are relatively prime and $a, b, c$ are either 1 or -1 .

Thus all but a finite number of such $P_{1}$ and $P_{2}$ will have $M(P)<1.3$, and it is of interest to examine those with, say, $M(P) \leqslant 1.25$.

We examined all of these for $h \leqslant 25$. None had measures less than $\sigma_{1}$. Among the $P_{1}$ have been found the smallest known values of $M(P)$ for fixed $\nu(P) \geqslant 4$. For example, the following degree 36 polynomial has $\nu(P)=4$, and $M(P)=1.2294828102$ :

$$
\begin{aligned}
& P=\begin{array}{lllllllllllllllllllll}
1 & 1 & 1 & 0 & 0 & -1 & -1 & -1 & 0 & 1 & 1 & 1 & 0 & -1 & -2 & -1 & 0 & 1 & 1 & 1 & 0
\end{array} \\
& \begin{array}{llllllllllllllllllllllll}
-1 & -2 & -1 & 0 & 1 & 1 & 1 & 0 & -1 & -1 & -1 & 0 & 0 & 1 & 1 & 1 .
\end{array} \text {. }
\end{aligned}
$$

This is a factor of $x^{44}+x^{35}-x^{31}-x^{22}-x^{13}+x^{9}+1$.

Another more extensive family which was investigated for $h \leqslant 20$ is the set of polynomials of the form

$$
P(x)=\left(x^{2 h+1}-x^{2 h-k}-x^{h+s+1}+x^{h-s}+x^{k+1}-1\right) /(x-1) .
$$

Since $L_{2}(P(x)(x-1))=6^{1 / 2}$, these all have $M(P) \leqslant 1+2^{1 / 2}$ by Gonçalves' inequality $(16)$. All known small Salem numbers $(\nu(P)=1)$ are measures of members of this family [2].

6. Lists of Polynomials. The composition of the following tables is described in Section 4. For degrees $\geqslant 18$, only the first half of the polynomial is listed, as in [2]. Only one of the polynomials listed is reducible, namely $\begin{array}{llllllllll}1 & 1 & -1 & -3 & -1 & 1 & 1=\end{array}$ $\left(\begin{array}{llll}1 & 0 & -1 & -1\end{array}\right)\left(\begin{array}{llll}1 & 1 & 0 & -1\end{array}\right)$, as discussed in Section 4 . In case $n \geqslant 18, M(P) \leqslant 1.27$ and $H(P)>1$, the number in parentheses following the degree is the smallest degree of a reciprocal $Q$ of even degree with $H(Q)=1$ of which $P$ is a factor. 
TABLE 1

All irreducible reciprocal polynomials $P$ of degree $n$ and measure $M(P) \leqslant M_{0}$, for $4 \leqslant n \leqslant 16$.

$$
\mathrm{n}=4, \mathrm{M}_{0}=2
$$

Measure

1.7220838057 1.8832035059

$$
\mathrm{n}=6, \mathrm{M}_{0}=2
$$

Measure

\subsection{9} 1. 5061356796 $1.55603 C 1913$ 1. 5823471837 1.6355731299 1.7467934983 1. 7548776662 1.7769445842

1. 7816435986

1. 7942797448

1.8310758251

1. 8392867552

1. 8548181172 1.9468562683 1.9635536390 1.9748187083 1. SE77931668 1. 9962080000
Polynomial

$\begin{array}{lllll}1 & 1 & -1 & 1 & 1\end{array}$

12121

Polynomial

$$
n=8, M_{0}=1.75
$$

Measure

1.2806381563

1.3599997117

1. 3672228038

1.3700868743

1. 4250052678

1.4575874796

1.4762823965

$1.4874 E 75383$

1.5230602489

1.53SE 181220

1. 54719 E 656

1.5682443656

1. $T C 54458288$

1.6185305986

1.6241475659

1.6403340873

1.6575497181

1. $661047762 \mathrm{C}$

1.6847101527

$1.685 C 319934$

1.6935073826

1. $7 C 42442644$

1. 7467934583
Polynomial

$\begin{array}{llllllllll}1 & C & 0 & 1 & -1 & 1 & 0 & 0 & 1\end{array}$

$\begin{array}{lllllllll}1 & 1 & 1 & 2 & 1 & 2 & 1 & 1 & 1\end{array}$

$\begin{array}{lllllllll}1 & 0 & 0 & 1 & 1 & 1 & 0 & 0 & 1\end{array}$

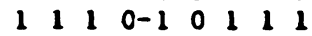

$\begin{array}{lllllllll}1 & 1 & 0 & 1 & 1 & 1 & 0 & 1 & 1\end{array}$

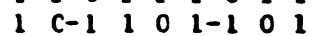

$\begin{array}{lllllllll}1 & 2 & 2 & 1 & 1 & 1 & 2 & 2 & 1\end{array}$

$\begin{array}{lllllllll}1 & 1 & 1 & 0 & 1 & 0 & 1 & 1 & 1\end{array}$

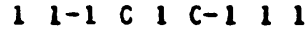

$\begin{array}{lllllllll}1 & 1 & 2 & 3 & 3 & 3 & 2 & 1 & 1\end{array}$

$\begin{array}{lllllllll}1 & 2 & 2 & 3 & 3 & 2 & 2\end{array}$

$\begin{array}{lllllllll}1 & 1 & 1 & 2 & 3 & 2 & 1 & 1 & 1\end{array}$

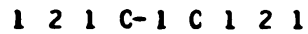

$\begin{array}{lllllllll}1 & 1 & 2 & 1 & 1 & 1 & 2 & 1 & 1\end{array}$

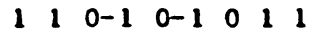

$\begin{array}{lllllllll}1 & 0 & -2 & 1 & 1 & 1-2 & 0 & 1\end{array}$

$\begin{array}{lllllllll}1 & 0 & 1 & 1 & 2 & 1 & 1 & 0 & 1\end{array}$

$\begin{array}{lllllllll}1 & 2 & 1 & 1 & 1 & 1 & 1 & 2 & 1\end{array}$

$\begin{array}{llllllll}1 & 1-1 & 0 & 0 & 0 & -1 & 1 & 1\end{array}$

$\begin{array}{llllllllll}1 & 1 & 1-1-2 & -1 & 1 & 1 & 1\end{array}$

$\begin{array}{llllllll}1 & 1 & 0 & 1-1 & 1 & 0 & 1 & 1\end{array}$

$\begin{array}{lllllllll}1 & 0 & 1 & 1 & -1 & 1 & 1 & 0 & 1\end{array}$

$11-1-1 \quad 1-1-1 \quad 1$ 
TABLE 1 (continued)

$$
\mathrm{n}=10, \mathrm{M}_{0}=1.5
$$

Measure

Polynomial

$1.17628 C 8183$

$1.21 \in 291 \in E 11$

1.2303914344

1. 2612305611

1.2672338594

1. $28358236 \mathrm{C6}$

1. 2934859531

1. $364 \$ 637617$

1.3373132102

1.3509863377

1.3619551623

1.3689986768

1. $3 E 3 \in 365634$

1.3984561816

$1.42599251 \mathrm{C7}$

$1.43100 \operatorname{c55} 51$

1. 4314046376

1.4484230402

1.4723531176

1.4723872015

1.4796086733

1. $4804088 C 56$

1.4824156382

1.4938778456
$1 \quad 10-1-1-1-1-10011$

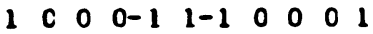

$\begin{array}{lllllllllll}1 & 0 & 0 & 1 & 0 & 1 & 0 & 1 & 0 & 0 & 1\end{array}$

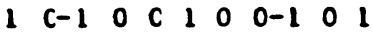

$\begin{array}{lllllllllll}1 & 0 & 1 & 1 & 0 & 1 & 0 & 1 & 1 & 0 & 1\end{array}$

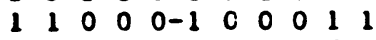

$\begin{array}{lllllllll}1 & 0 & -1 & 1 & 0-1 & 0 & 1-1 & 0 & 1\end{array}$

$\begin{array}{lllllllll}1 & 1 & 0-1 & 0 & 0 & 0-1 & 0 & 1 & 1\end{array}$

$\begin{array}{lllllllllll}1 & 1 & 0 & 0 & 0 & 1 & 0 & 0 & 0 & 1 & 1\end{array}$

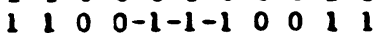

$\begin{array}{lllllllllll}1 & 0 & 0 & 0 & 1 & 1 & 1 & 0 & 0 & 0 & 1\end{array}$

$\begin{array}{llllllllllll}1 & 0 & 0 & 1 & 0 & -1 & 0 & 1 & 0 & 0 & 1\end{array}$

$\begin{array}{lllllllllll}1 & 1 & 0 & 1 & 1 & 1 & 1 & 1 & 0 & 1 & 1\end{array}$

1122232221

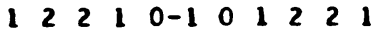

$\begin{array}{lllllllll}1 & 1-1-1 & 0 & 1 & 0 & -1-1 & 1 & 1\end{array}$

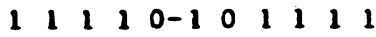

$\begin{array}{lllllllllll}1 & 2 & 2 & 2 & 1 & 1 & 1 & 2 & 2 & 2 & 1\end{array}$

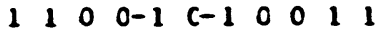

$\begin{array}{lllllllllll}1 & 0 & 1 & 1 & 1 & 0 & 1 & 1 & 1 & 0 & 1\end{array}$

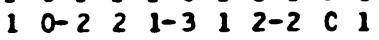

$\begin{array}{lllllllllll}1 & 1 & 2 & 3 & 3 & 3 & 3 & 3 & 2 & 1 & 1\end{array}$

$\begin{array}{lllllllllll}1 & 1 & 1 & C & 1 & 0 & 1 & 0 & 1 & 1 & 1\end{array}$

$\begin{array}{llllllll}1 & 0-1 & 1 & 1-2 & 1 & 1-1 & 0 & 1\end{array}$

$\mathrm{n}=12, \mathrm{M}_{\mathrm{o}}=1.4$

Measure

1.2277855587

1.2407264237

1. 2510466172

1. 2643938547

$1.27281 \varepsilon 3651$

1.3019549435

1. $3 C 22688051$

1. 3644325627

1.3101213237

1.3159144319

1. 3221661921

1. 3349522567

1.3434948981

1. 3702684012

1.3776747894

1. 3877955034

1.3887530310

1.3977398823
Polynomial

$1110-1-1-1-1-1 \quad 0 \quad 1111$

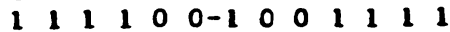

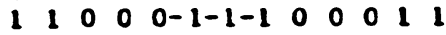

$\begin{array}{llllllllllll}1 & 0 & 1 & 0 & 0 & 1-1 & 1 & 0 & 0 & 1 & 0 & 1\end{array}$

$\begin{array}{lllllllllllll}1 & 0 & 1 & 1 & 1 & 2 & 1 & 2 & 1 & 1 & 1 & 0 & 1\end{array}$

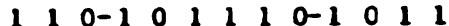

$\begin{array}{lllllllllllll}1 & 1 & 0 & 0 & 0 & 1 & 1 & 1 & 0 & 0 & 0 & 1 & 1\end{array}$

$\begin{array}{lllllllllll}1 & 0 & 0 & 1-1-1 & 1-1-1 & 1 & 0 & 0 & 1\end{array}$

$\begin{array}{lllllllllllll}1 & 2 & 2 & 1 & 1 & 1 & 1 & 1 & 1 & 1 & 2 & 2 & 1\end{array}$

$\begin{array}{llllllllllll}1 & 0 & 0 & 0 & -1 & 1-1 & 1-1 & 0 & 0 & 0 & 1\end{array}$

$\begin{array}{lllllllllllll}1 & 0 & 0 & 0 & 0 & 1 & 1 & 1 & 0 & 0 & 0 & 0 & 1\end{array}$

$\begin{array}{lllllllllllll}1 & 1 & 0 & 0 & 0 & 0 & 1 & 0 & 0 & 0 & 0 & 1 & 1\end{array}$

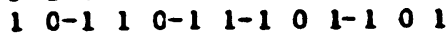

$\begin{array}{llllllllllll}1 & 0 & -1 & 1 & 0 & 0 & 0 & 0 & 0 & 1-1 & 0 & 1\end{array}$

$\begin{array}{llllllllllllll}1 & 0 & 0 & 0 & 0 & 1-1 & 1 & 0 & 0-1 & 0 & 1\end{array}$

$\begin{array}{lllllllllllll}1 & 0 & 1 & 0 & 1 & 1 & 0 & 1 & 1 & 0 & 1 & 0 & 1\end{array}$

$\begin{array}{lllllllllllll}1 & 0 & 0 & 0 & 1 & 1-1 & 1 & 1 & 0 & 0 & 0 & 1\end{array}$

1222333332221 
TABLE 1 (continued)

$$
\mathrm{n}=14, \mathrm{M}_{\mathrm{o}}=1.33
$$

Measure $\quad v$

Polynomial

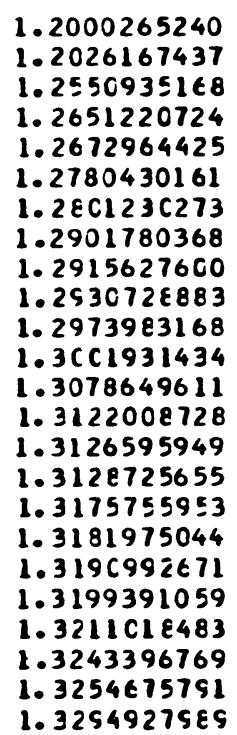

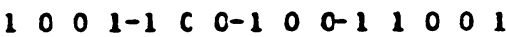

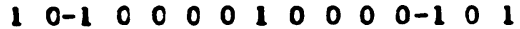

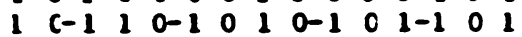
$\begin{array}{lllllllllllllll}1 & 0 & 0 & 1 & 1 & 1 & 1 & 1 & 1 & 1 & 1 & 1 & 0 & 0 & 1\end{array}$

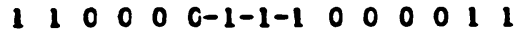
$1 \quad 1 \quad 1 \quad 000-1-1-1-1-1 \quad 0 \quad 0 \quad 1 \quad 11$

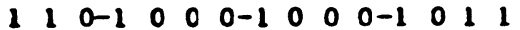

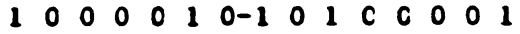
$1110-1-1-1-1-1-1-1 \quad 0 \quad 1 \quad 11$

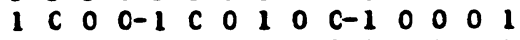

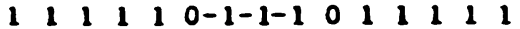
$\begin{array}{lllllllllllllll}1 & 1 & 0 & 0 & 0 & C & 1 & 1 & 1 & 0 & 0 & 0 & 0 & 1 & 1\end{array}$

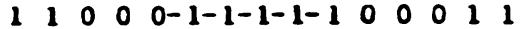

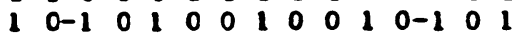
$\begin{array}{llllllllllllll}1 & 0 & 0 & 1 & c-1 & c & 0 & 0 & -1 & 0 & 1 & 0 & 0 & 1\end{array}$ $\begin{array}{lllllllllllllll}1 & 0 & 0 & 0 & 0 & 1 & 0 & 1 & 0 & 1 & 0 & 0 & 0 & 0 & 1\end{array}$ $\begin{array}{llllllllllllll}1 & 0 & 1 & 0 & 0 & 1-1 & 1-1 & 1 & 0 & 0 & 1 & 0 & 1\end{array}$ $\begin{array}{lllllllllllllll}1 & 1 & 0 & 1 & 1 & 0 & 0 & 1 & 0 & C & 1 & 1 & 0 & 1 & 1\end{array}$

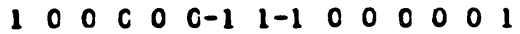
$\begin{array}{lllllllllllllll}1 & 0 & 0 & 1 & 0 & 0 & 0 & -1 & 0 & 0 & 0 & 1 & 0 & 0 & 1\end{array}$

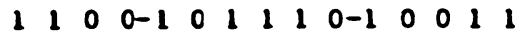
$\begin{array}{llllllllllllll}1 & 1 & 0-1-1-1 & 0 & 1 & 0-1-1-1 & 0 & 1 & 1\end{array}$ $\begin{array}{lllllllllllllll}1 & 0 & 0 & 1 & 1 & 0 & 0 & 1 & 0 & 0 & 1 & 1 & 0 & 0 & 1\end{array}$ $\begin{array}{lllllllllllllll}1 & 0 & 1 & 0 & 0 & 1 & 0 & 1 & 0 & 1 & 0 & 0 & 1 & 0 & 1\end{array}$

$\mathrm{n}=16, \mathrm{M}_{\mathrm{O}}=1.3$

Measure

$v$

Polynomial

1. 2242789072 $1.2352567 C 56$ 1.2363175318 1.2434776187 1. $248 C 136 C 83$ 1.2517324113 1. $252 \varepsilon 286630$ 1.2573166256 1. 2 EEE 775265 1.2813411294 $1.28959 C 1 C 23$ 1.2934931125 1.2570647745 1. $29803 \check{2543}$

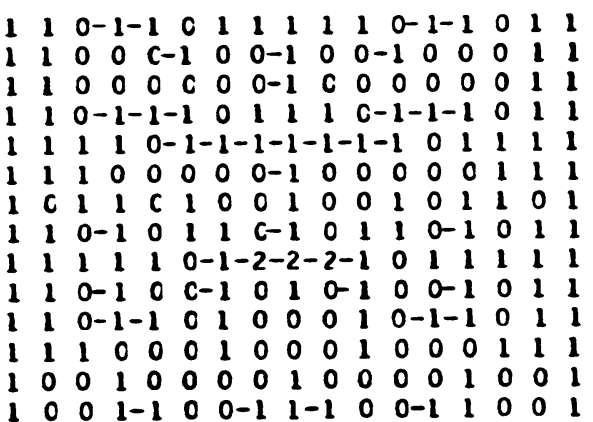


TABLE 2

All irreducible reciprocal polynomials of degree $n, 18 \leqslant n \leqslant 26$,

which divide a reciprocal polynomial of height 1

and even degree at most 26, having measure at most 1.27 .

\begin{tabular}{|c|c|c|c|}
\hline $\mathbf{n}$ & Measure & $v$ & First half of polynomial \\
\hline $\begin{array}{l}18 \\
18 \\
18 \\
18 \\
18 \\
18 \\
18 \\
18 \\
18 \\
18 \\
18\end{array}$ & $\begin{array}{l}1.1883681475 \\
1.2013961862 \\
1.219446 E 759 \\
1.2197208590 \\
1.2255034241 \\
1.2313427760 \\
1.24 C 77 C 6350 \\
1.244617 C 550 \\
1.2527759374 \\
1.2562211544 \\
1.2663343250\end{array}$ & $\begin{array}{l}1 \\
2 \\
3 \\
1 \\
2 \\
3 \\
2 \\
3 \\
1 \\
1 \\
3\end{array}$ & $\begin{array}{rrrrrrrrrr}1 & 1 & 1 & 1 & 0 & 0-1-1-1-1 \\
1 & 1 & 1 & 0 & 0-1 & 0 & -1 & 0-1 \\
1 & 1 & 1 & 0-1 & -1 & -1 & 0 & 0 & 1 \\
1 & 1 & 0 & C & C & C & 0 & 0-1-1 \\
1 & 1 & 0 & 0 & 1 & 0-1 & 0 & 0-1 \\
1 & C & 0 & C-1 & 1 & 0 & 0 & 0-1 \\
1 & 0 & 1 & 0 & 1 & 0 & 0 & 1-1 & 1 \\
1 & 0 & 1 & 0 & 1 \\
1 & 0 & -1 & 0 & 1 & C-1 & 0 & 0 & 1 \\
1 & 0 & 0 & 0 & 0 & 0-1 & 1-1 & 1 \\
1 & 1 & 0 & 0-1 & -1 & 0 & 0 & 0 & 1 \\
1 & 1 & 0 & 0 & 0-1 & 1-1 & 0 & 0-1\end{array}$ \\
\hline
\end{tabular}

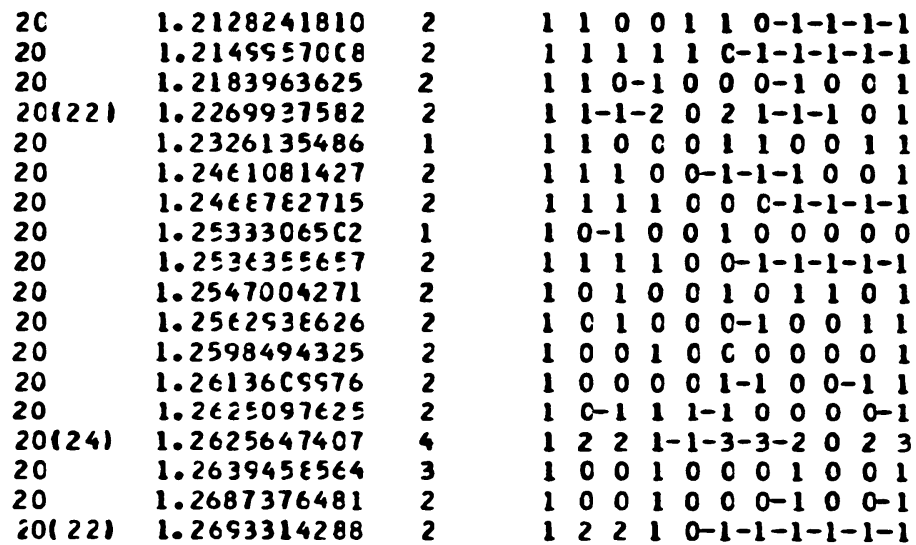

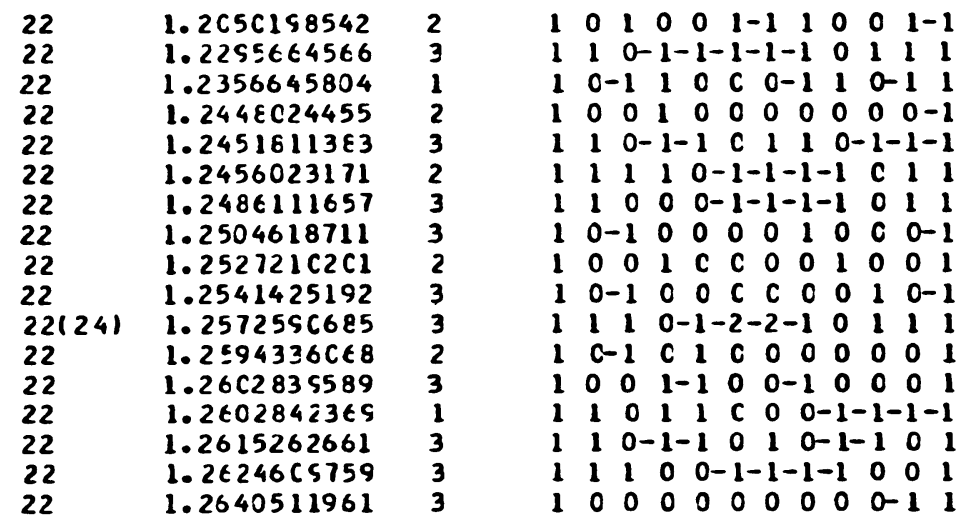




\section{TABLE 2 (continued)}

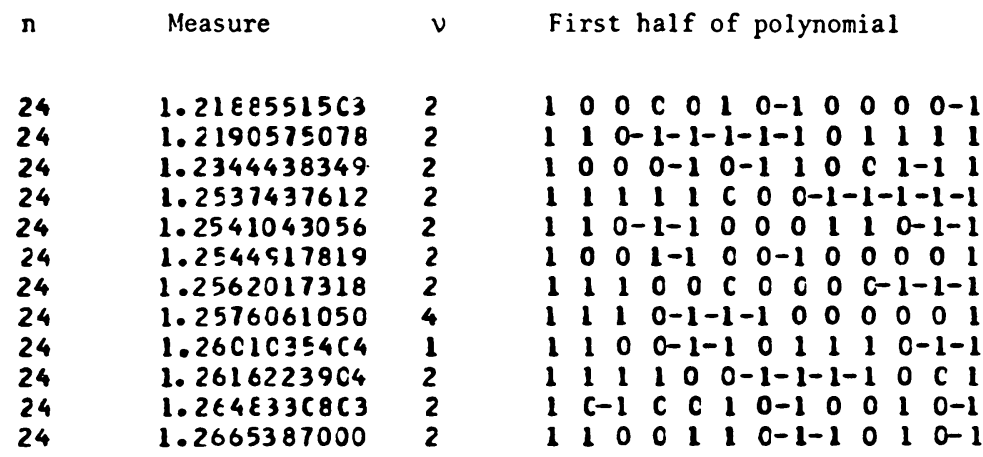

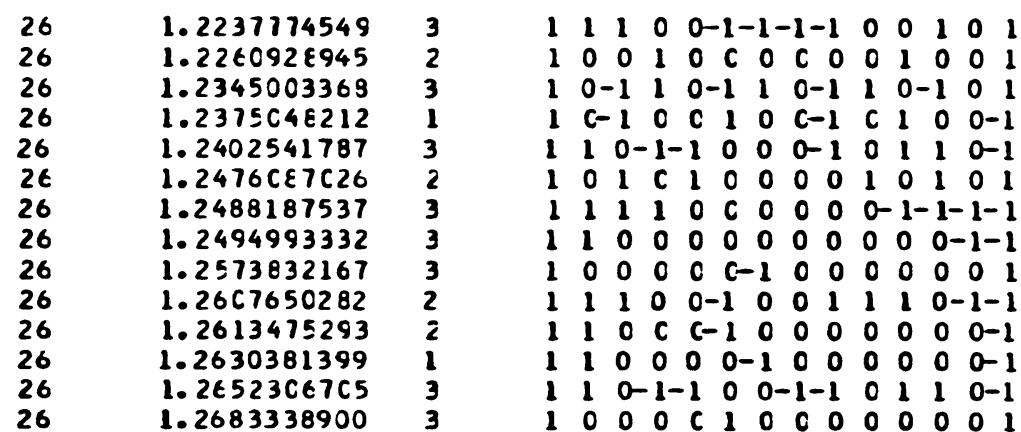

Department of Mathematics

The University of British Columbia

Vancouver, B. C., Canada V6T 1W5

1. E. H. BAREISS, "Resultant procedure and the mechanization of the Graeffe process," J. Assoc. Comput. Mach., v. 7, 1960, pp. 346-386.

2. D. W. BOYD, "Small Salem numbers," Duke Math. J., v. 44, 1977, pp. 315-327.

3. D. W. BOYD, "Variations on a theme of Kronecker," Canad. Math. Bull., v. 21, 1978, pp. $129-133$.

4. D. W. BOYD, "Pisot numbers and the width of meromorphic functions." (Privately circulated manuscript.)

5. E. DOBROWOLSKI, "On a question of Lehmer and the number of irreducible factors of a polynomial," Acta Arith., v. 34, 1979, pp. 391-401.

6. R. L. DUNCAN, "Some inequalities for polynomials," Amer. Math. Monthly, v. 73, 1966, pp. 58-59.

7. D. H. LEHMER, "Factorization of certain cyclotomic functions," Ann. of Math. (2), v. 34,1933 , pp. 461-479.

8. K. MAHLER, Lectures on Transcendental Numbers, Lecture Notes in Math., Vol. 546, Springer-Verlag, Berlin and New York, 1976.

9. M. MARDEN, Geometry of Polynomials, 2nd ed., Math. Surveys, no. 3, Amer. Math. Soc., Providence, R. I., 1966.

10. D. S. MITRINOVIC, Analytic Inequalities, Springer-Verlag, Berlin and New York, 1970.

11. A. OSTROWSKI, "On an inequality of J. Vincente Gonçalves," Univ. Lisboa Rev. Fac. Ciênc. $A$ (2), v. 8, 1960, pp. 115-119.

12. C. J. SMYTH, "On the product of the conjugates outside the unit circle of an algebraic integer," Bull. London Math. Soc., v. 3, 1971, pp. 169-175.

13. C. L. STEWART, "On a theorem of Kronecker and a related question of Lehmer," Séminaire de Theorie des Nombres, Bordeaux, 1977/78, no. 7 (11 pp.). 